\title{
THE EVALUATION OF KIEL BONE IN SPINAL FUSIONS
}

\author{
G. N. McMURRAY \\ From the Withers Orthopaedic Centre, Belfast
}

\begin{abstract}
The clinical, radiological and histological information on the fate of Kiel bone implants for anterior spinal fusion in four patients was evaluated. These implants were found to be unsatisfactory and further operations were required. Biopsies of the grafts showed that the Kiel bone was invaded by fibrous tissue but that it did not ossify or become incorporated into the underlying bone. However, it might provide temporary support to the grafted area. These findings indicate that the use of Kiel grafts does not seem to be justified in current orthopaedic practice.
\end{abstract}

The use of lyophilised bone from calves as a heterograft was introduced by Maatz and Bauermeister in 1957 as an alternative to autografts and freeze-dried homografts which were then in general use. Lyophilised bone is prepared from the bone of young calves or pigs and consists of fibres and crystallites with a minimal residual of protein arranged biochemically in a scaffold formation. To prevent thermal denaturing the temperature used in the preparation of this bone is less than 34 degrees Celsius.

The product is known as Kiel bone in Germany and Great Britain; the nearest equivalent in the USA is "Boplant" which is prepared in a different way and has a different ultrastructure from the Kiel bone. The Kiel bone was shown to possess no antigenicity (AMA 1966) and serial radiographs have shown that it is incorporated into the recipient site by a process of creeping substitution from surrounding bone (Fuchs 1964). No definite contra-indications to the use of such bone have been reported, although there has been some degree of rejection and absorption of the graft in patients where the graft was very large, where inflammation was present, where haematomata had formed, or where the recipient was pyrexial. However, there is a contraindication in patients who have an allergy to horse serum since Kiel bone contains two similar but weak antigens. A reduction in the operating time is possible with Kiel bone since no bone grafts are required. Consequently, there are no painful donor sites which are often affected by formation of haematomata and infection of the wound.

After the introduction of Kiel bone it was used widely in virtually every possible graft site with variable success. Jackson (1971) used Kiel bone in spinal fusions to prevent delayed union caused by the effect of rib grafts as reported by Hodgson and Stock (1960). Jackson noted that the Kiel bone caused a bridge of autogenous bone to form from the surrounding auto- genous bone and encompass the defect. This was attributed to either a mild inflammatory response or to an auto-immune response. He advised using chips of autogenous bone mixed with Kiel grafts for large defects. In this situation the Kiel graft acted as a scaffold and also reduced the amount of autogenous bone required which is an important consideration in children.

The results using Kiel bone for cervical fusions at the C5-6 and C6-7 levels in a series of 263 patients were found by Taheri (1972) to be satisfactory after clinical and radiological assessment. Biopsies were not taken in this series. The disappearance of the halo seen around the graft on early anteroposterior radiographs taken after operation was used to indicate fusion of the graft. This halo was thought to be caused by a layer of uncalcified osteoid material encompassing the graft.

In 1975 Ramani, Kalbag and Sengupta used lyophilised bone in 73 cervical disc spaces and made a follow-up of their patients clinically and radiologically for between two and five years. Although the clinical results were satisfactory, no true radiological union could be demonstrated. The halo reported by Taheri (1972) was seen in anteroposterior radiographs, but in cases where it had disappeared from anteroposterior views, it could still be demonstrated using tomography.

\section{METHODS AND MATERIAL}

The literature does not contain reports of biopsies taken from implanted lyophilised bone at intervals after its use in anterior spinal fusions. In this series, three patients had an anterior fusion of the lumbar spine for intractable backache. The fourth patient (a three and a half-yearold boy) had a congenital thoracic kyphosis and developed a paraplegia.

In all four patients the material used for grafting was lyophilised bone. The clinical results were unsatisfactory and further operations were required at varying 


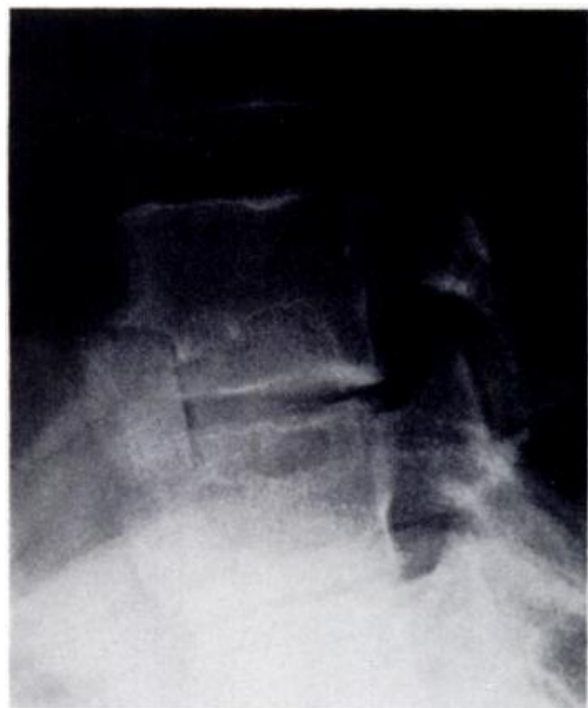

Fig. 1

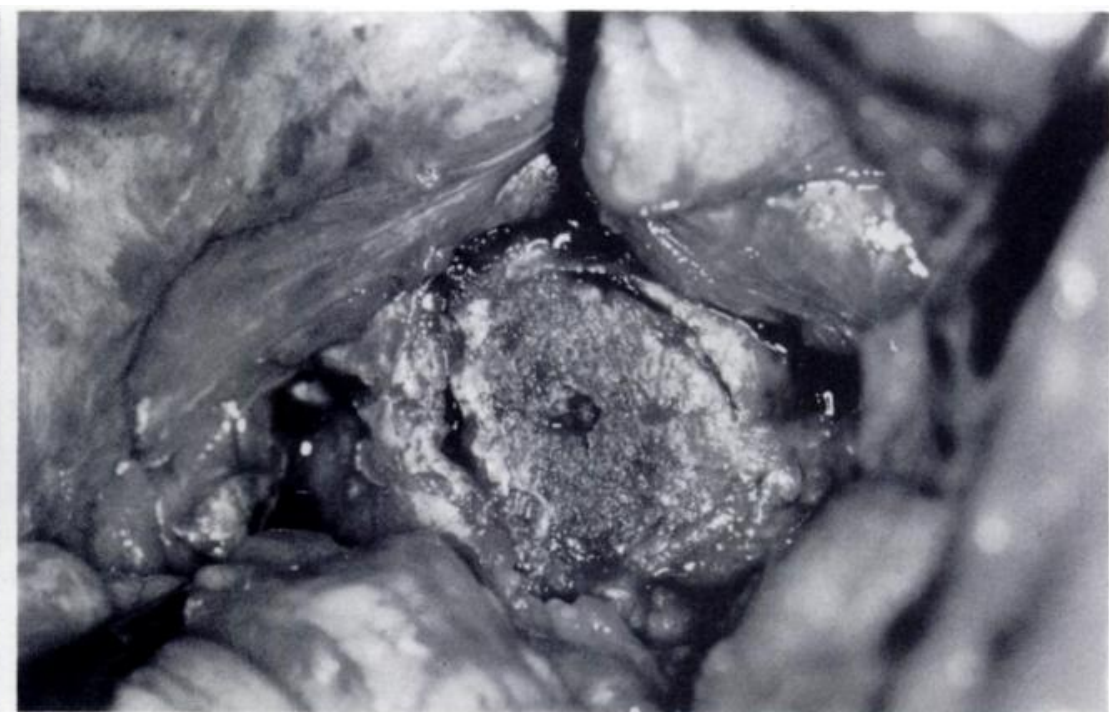

Fig. 2

Case 2. Figure 1-Radiograph of a Kiel graft three months after implantation. Figure 2-Removal of the Kiel dowel 17 months after implantation.

intervals after the initial operation in all cases before a satisfactory result was obtained. This enabled a chronological series of biopsies from Kiel bone implants to be obtained.

\section{CASE HISTORIES}

Case 1. A woman aged 36 years with a six-year-history of low backache was unrelieved by prolonged conservative therapy. In January 1966, anterior spinal fusions at the L4-5 and L5-S1 levels were performed using lyophilised bone. In March 1966, laparotomy was necessary for abdominal pain and a cyst involving the nght fallopian tube was found. It was removed along with an atrophic right ovary. A biopsy of the graft was performed eight weeks after the date of insertion. Histology of the Kiel bone revealed fibroblastic reaction. Subsequent radiographs showed no evidence of fusion although the patient was free from pain. In October 1968, further backache was reported with restricted straight leg raising. Radiographs showed fusion of L5-S1, the existence of a halo at L4-5, and a possible pseudarthrosis between the graft and the L5 vertebra. Re-exploration of her back in January 1969 revealed that there was a fibrous tissue wall around the graft. A biopsy of the graft at L4- 5 was performed but the graft was not removed. In October 1969, her backache recurred. A posterior spinal fusion using autogenous bone was carried out; the results were satisfactory and she was finally discharged in 1971 free from symptoms

Case 2. A women aged 44 years with a 10-year-history of backache had a laminectomy and fenestration at L4-5 in 1963. Two years later further backache was reported. In March 1968, an anterior fusion at L3-4 and L4-5 was carried out using one Kiel implant mixed with chips of autogenous bone at L3-4 and two Kiel implants only at L4-5. At review, in June 1968, there was radiographic evidence of fusion of the grafts and no haloes were noted (Fig. 1).

In July 1969, she had further pain; L4- 5 was radiologically fused but L3-4 appeared fused by a bridge of autogenous bone anterior to the graft. Radiographic screening showed L3-4 to be unstable. At operation in November 1969 the Kiel dowels at L4-5 were found to be mobile with a fibrous pseudarthrosis. These dowels were easily removed 17 months after implantation (Fig. 2). There was doubtful mobility at L3-4 and a biopsy of the graft was performed when L3 to 5 were re-grafted with autogenous bone. The patient was lost to review until 1974 when she presented with an abscess on the left iliac crest and also on the left psoas. A sinogram indicated a tract to the L4- 5 level possibly caused by rejection of the Kiel bone. In 1975 further discharge from the sinus was treated conservatively.
Biopsies were taken 17 months after implantation. The Kiel bone graft dowel at the L4-5 level consisted of dead Kiel bone with amorphous debris in spaces between the trabeculae. At the margins, there was some invasion of the trabeculae by dense fibrous tissue with partial removal of Kiel bone. Foreign-body giant-cell formation was noted but there was no evidence of invasion of the Kiel bone by autogenous bone (Fig. 3). Biopsy from L3-4 showed mainly autogenous bone which was entirely dead with minimal reactive fibrosis and a few small mononuclear cells. The junction between the Kiel bone and the vertebra showed a dense fibrous invasion of Kiel bone trabeculae at the margins of the specimen with central necrosis

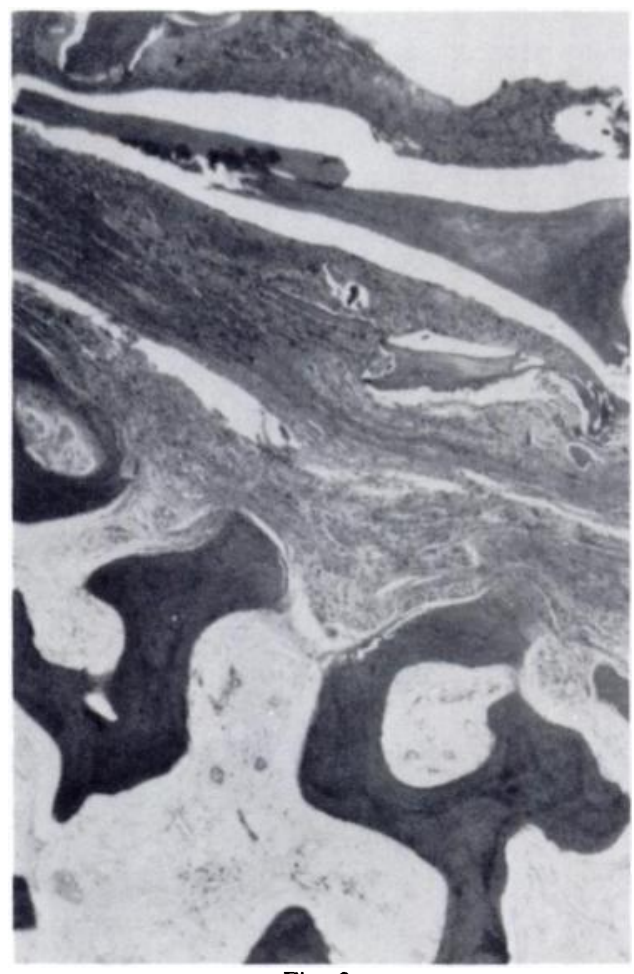

Fig. 3

Histological section showing fibrous tissue invasion of the Kiel graft 17 months after implantation. There is no evidence of invasion of the Kiel bone by autogenous bone 
indicative of previous fibrosis in the central area. A mild foreign-body giant-cell reaction to the fibrosis was present at the margins but the majority of the bone was dead. There was no evidence of autogenous bone ingrowth into the Kiel bone. Isolated fragments of Kiel bone showed a dense surrounding fibrous tissue reaction with foreign-body giant-cell systems present.

Case 3. A woman aged 31 years presented with a history of intractable lumbosacral pain after a fall. There was no relief of this pain despite prolonged conservative treatment. In April 1966, anterior spinal fusion was performed at the L5-S1 level using both Kiel bone and iliac crest bone. In May 1968, re-exploration for persistent backache and non-union of the graft was made (Fig. 4). The Kiel graft was removed

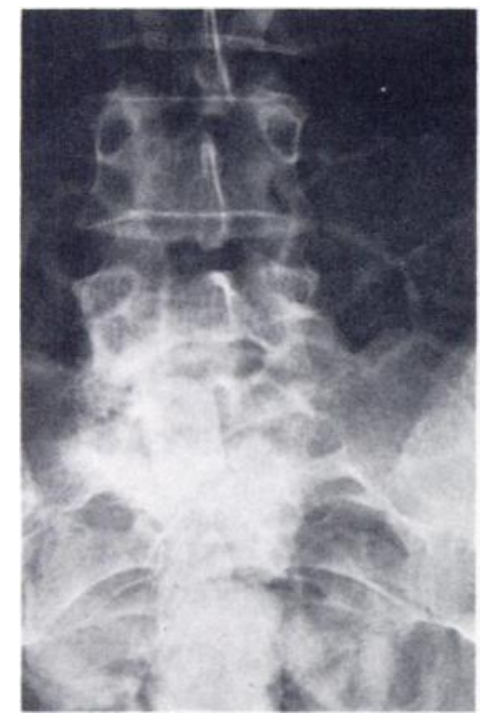

Fig. 4

Case 3. Radiograph showing the halo caused by dense fibrosis around the Kiel graft

and found to have a fibrous capsule around it. The area was re-grafted using autogenous bone; the subsequent result was satisfactory. Biopsy 25 months after implantation showed no evidence of autogenous bone ingrowth. Dense surrounding fibrosis was seen with no evidence of infection (Fig. 5).

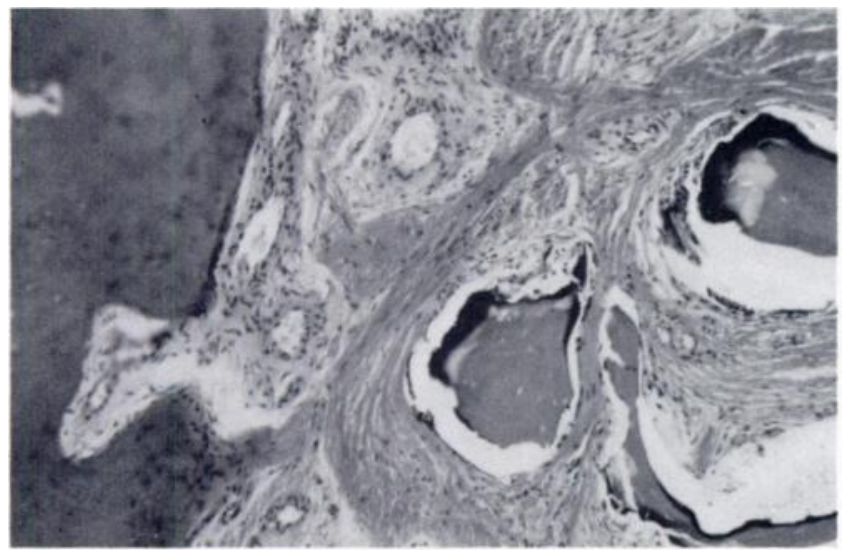

Fig. 5

Histological section of the Kiel graft in Case 3. There is dense fibrosis present with no evidence of autogenous bone ingrowth.

Case 4. A boy aged three and a half years presented with congenital kyphosis and the signs of the onset of paraplegia. In 1975 posterolateral decompression and spinal osteotomy with the insertion of a cube of Kiel bone were performed. Six weeks later there was radiological evidence of possible dislocation of the graft on to the spinal cord; further decompression and homogenous (maternal) bone grafting were necessary. Biopsy of the Kiel graft six weeks after insertion indicated mononuclear infiltration, and the formation of dense collagen and fibrous tissue which had invaded the bone trabeculae. A few polymorphs were seen at the edges of the specimen but there was no evidence of infection. There was no histological evidence of dislocation of the graft, judging by the degree of organisation and fibrosis around it, and no evidence of graft substitution by autogenous bone.

\section{DISCUSSION}

These case histories provide clinical, radiological and histological information on the fate of Kiel bone implants between six weeks and three years after insertion. Clinically our results were unsatisfactory since all four patients required autogenous bone grafting before satisfactory results were obtained. There are conflicting opinions concerning the halo seen around the graft on radiographs and its possible later disappearance (Taheri 1972; Ramani et al. 1975). This makes interpretation of the radiographic evidence for fusion of the Kiel graft with the bone speculative and unreliable. It is suggested that the halo may simply represent the presence of a dense fibrosis around the implant since the histological studies provided no evidence of autogenous bone invasion of the graft.

Macroscopically it appears that the Kiel graft has been incorporated into the bone at operation, but this is misleading and contrasts with subsequent histological reports of the area. Roaf (1964) reported a series of two-stage spinal fusions undertaken for the treatment of scoliosis and tuberculosis using both Kiel bone and autografts. Although the area appeared to be a solid sheet of bone without formation of a pseudarthrosis at the second operation three to four months later, biopsies revealed areas of inert bone and the histology was so featureless that the original type of material could not be determined.

Histological analysis of the biopsy material suggests that lyophilised bone is surrounded by a capsule of fibrous tissue at a surprisingly early time after implantation and it may be the presence of fibrosis which ultimately determines the success of the graft.

In Case 4, where the biopsy was taken six weeks after implantation, a substantial degree of fibrosis was already present with invasion by fibroblasts of the lacunae of the Kiel bone. Formation of collagen also occurred, indicating maturation of the fibroblasts.

In Case 1, where the biopsy was taken two months after implantation, evidence of dense fibrosis was present. There was formation of a pseudarthrosis which would suggest that movement of the graft had occurred. Incorporation of the graft into the surrounding bone was most unlikely to occur. In addition, mononuclear and plasma cells were found at the edges of the graft. This might indicate that the Kiel bone is not entirely inert as has been originally claimed (AMA 1966) or it may 
merely represent a non-specific reaction to tissue injury during operation.

Later biopsies (Case 2), taken 17 months after implantation again confirmed the presence of fibrosis, dead bone and invasion of the graft by autogenous bone. Biopsies of Cases 1 and 3 both show a dense fibrosis around the graft with some extension of fibroblasts into the graft trabeculae. In these later biopsies, there was fragmentation of the Kiel bone and formation of dense collagen fibres which in some places had undergone necrosis. A few foreign-body giant-cell systems were noted in all the patients. These findings suggest that Kiel bone merely provides a scaffold upon which fibrous tissue can grow and mature to collagen and finally necrose once it has strangulated its blood supply. However, the presence of fibrous tissue and collagen may give some structural support to the area.

There was no histological evidence of incorporation of the Kiel bone into the autogenous bone in which it had been implanted. Osteoconduction, as described by Fuchs (1964) was nat observed. This was unexpected since the grafts had been inserted into the vascular spongy bone of the vertebral column which is one of the most favourable areas for bone grafts.

The effect of spinal movement upon the grafts is speculative. The formation of dense fibrous tissue and collagen may have been partially excited by movement. Further evidence of movement may be indicated by the presence of pseudarthroses found in several of our histological specimens. The attachment of the fibroblasts and collagen fibres to the Kiel bone may not be secure and while the graft and the fibrosis around it may provide initial stabilisation of the area the subsequent fragmentation of the Kiel bone and collagen may be the cause of recurrent backache in three of the patients.

The mechanical stresses on a Kiel graft in the spine are probably greater than those which a Kiel graft in a long bone would be exposed to since a limb can be more effectively immobilised than the spine. There could be a species-specific reaction to Kiel bone. The early experimental work was performed on rabbits and dogs in which the size of implants used had been relatively small whereas those used in man have been much larger, even after consideration of the relative differences in size.

In conclusion Kiel bone is not comparable with autogenous bone for the purpose of grafting. No evidence of osteoconduction was found indicating incorporation of the Kiel bone into the recipient sites and it seems that Kiel bone acts more like a virtually inert foreign body until the surrounding fibrosis becomes so dense that collagen necrosis ensues. In situations where the Kiel graft is under stress, it may undergo fragmentation with occasional inflammatory sequestration. Where Kiel bone is used in large cavities, it may help by providing a scaffold over which autogenous bone may spread. The dense collagen and fibrous tissue evoked by Kiel bone may also provide a temporary support to the grafted area but in time this support will be the underlying cause of the final failure of the Kiel graft. The use of Kiel grafts therefore cannot be justified in current orthopaedic practice, except perhaps in situations where the cavity to be grafted is large and the supply of iliac crest bone is limited.

I acknowledge the help of Mr N. S. Martin, Senior Consultant Orthopaedic Surgeon at Musgrave Park Hospital, in the preparation of this paper and for his permission to study the patients under his care. I also acknowledge the help with the histological reports given to me by Professor J. E. Morison.

\section{REFERENCES}

American Medical Association Council on Drugs. A new implant material for use in reconstructive surgical procedures: Surgibone (Boplant). JAMA 1966;195:951-2.

Fuchs G. Interrelation of bone graft chemistry and its fate. In: Symposium on bone grafing materials. Eastbourne: Armour Pharmaceuticals Ltd, 1964:5-19.

Hodgson AR, Stock FE. Anterior spine fusion for the treatment of tuberculosis of the spine: the operative findings and results in the first one hundred cases. J Bone Joint Surg [Am] 1960;42-A:295-310.

Jackson JW. Surgical approaches to the anterior aspect of the spinal column. Ann R Coll Surg Engl 1971;48:83-98.

Maatz R, Bauermeister A. A method of bone maceration. J Bone Joint Surg [Am] 1957;39-A:153-66.

Ramani PS, Kalbag RM, Sengupta RP. Cervical spinal interbody fusion with Kiel bone. Br J Surg 1975;62:147-50.

Roaf R. Deproteinised bone, its value heterologically and radiologically. In: Symposium on bone grafting materials. Eastbourne: Armour Pharmaceuticals Ltd, 1964:93-115.

Taheri ZE. Experience with calf bone in cervical interbody spinal fusion. J Neurosurg 1972;36:67-71. 